PROCEEDINGS OF THE

AMERICAN MATHEMATICAL SOCIETY

Volume 103, Number 1, May 1988

\title{
SPACES EVERY QUOTIENT OF WHICH IS METRIZABLE
}

\author{
A. J. JAYANTHAN AND V. KANNAN
}

(Communicated by Dennis Burke)

\begin{abstract}
We characterise those topological spaces for which every quotient image is metrizable. This supplements the earlier known results in this direction, in a fairly complete manner.
\end{abstract}

0. Introduction. Metrizability of quotients of metric spaces has been studied by many mathematicians $[\mathbf{1}, \mathbf{2}, \mathbf{6}, \mathbf{7}]$. In an elementary course in general topology we learn that every continuous image in Hausdorff space of a compact metric space is metrizable [8]. In [7], Willard proved that every closed continuous image of a metric space $X$ in Hausdorff space is metrizable if and only if the set of all limit points of $X$ is compact. We consider the following problem. What are all topological spaces every Hausdorff quotient space of which is metrizable? To our surprise, the condition on the space turns out to be the same as that of Willard. We prove that every quotient of a metric space $X$ in Hausdorff spaces is metrizable if and only if the set of all limit points of $X$ is compact. In the process we give plenty of equivalent conditions for this.

1. The main theorem. We first fix some notation and terminology. Let $X$ be a topological space. The set of all limit points of $X$ is denoted by $X^{1}$. By the term map we always mean a continuous map. Let $Y$ be any other topological space and $f: X \rightarrow Y$ be any map onto $Y$. We say that $f$ is compact-to-one (respectively finite-to-one, $n$-to-one) if $f^{-1}(y)$ is a compact (respectively a finite, having at most $n$ points) subset of $X$ for all points $y$ in $Y$, and in these cases $Y$ is called a compactto-one (respectively finite-to-one, $n$-to-one) image of $X$. For $f: X \rightarrow Y$ and $A \subset X$, we say that $A$ is saturated with respect to $f$, if $f^{-1}(f(a)) \subset A$ for all $a$ in $A$. All spaces considered in this article are assumed to be Hausdorff spaces and all maps are assumed to be continuous from Hausdorff spaces to Hausdorff spaces. A quotient space which is also a Hausdorff space is called a Hausdorff quotient space or simply a Hausdorff quotient. $\omega$ denotes the first infinite ordinal number and $\mathbf{N}$ denotes the set of all natural numbers.

EXAMPLE. We now see a simple example of a metric space which has a nonmetrizable quotient. Let $A=\left\{0,1, \frac{1}{2}, \frac{1}{3}, \ldots\right\}$. Define $X=\mathbf{N} \cup\{0\} \times A \subset \mathbf{R}^{2}$ and $Y=\{(x, y) \in A \times A: x=0$ implies $y=0\}$. Define a map $q: X \rightarrow Y$ as follows:

$$
q(x)= \begin{cases}(1 / n, 0) & \text { if } x \text { is }(0,1 / n) \text { or }(n, 0), \\ (1 / m, 1 / n) & \text { if } x \text { is }(m, 1 / n), m \geq 1, \\ (0,0) & \text { if } x \text { is }(0,0) .\end{cases}
$$

Received by the editors February 12, 1987.

1980 Mathematics Subject Classification (1985 Revision). Primary 54B15, 54E35.

Key words and phrases. Quotient spaces, metrizability.

The first author was supported by the Council of Scientific and Industrial Research, India. 
Give the usual topology for $X$ and the quotient topology induced by $q$ for $Y$. One can verify that $Y$ is not even first countable. $Y$ with this topology is denoted by $S_{2}$ throughout this article (see also [3]). We exploit properties of $S_{2}$ directly and indirectly at many places below.

A metric $d$ on a metric space $X$ is called normal if $d(A, B)=\inf \{d(a, b): a \in A$, $b \in B\}>0$ for every pair $A, B$ of disjoint closed subsets of $X$. The following theorem will be useful in the proof of our main theorem.

THEOREM 1.1 [4]. Suppose $X$ is a metric space. Then there exists an equivalent normal metric on $X$ if and only if $X^{1}$ is compact.

Now we prove the first set of equivalent forms to the metrizability of every Hausdorff quotient of a metric space. Hereafter we write simply "quotient" for Hausdorff quotient space.

THEOREM 1.2. Let $X$ be a metric space. Then the following conditions are equivalent:

(1) Every quotient of $X$ is metrizable.

(2) $X$ is the union of a compact subspace and a discrete subspace.

(3) $X^{1}$ is compact.

(4) $\omega^{2}$ cannot be embedded as a closed subspace in $X$.

PROOF. (1) $\Rightarrow(2)$. Suppose $X$ cannot be written as a union of a compact subspace and a discrete subspace. We know that $X \backslash X^{1}$ is a discrete subspace and hence $X^{1}$ is not compact. Choose a sequence $\left\{x_{n}\right\} \subset X^{1}$ such that $x_{m} \neq x_{n}$ for every $m \neq n$ and $\left\{x_{n}: n \in \mathbf{N}\right\}$ does not have a limit point in $X$. So $\left\{x_{n}: n \in \mathbf{N}\right\}$ is a closed subspace of $X$. Since $x_{1}$ is in $X^{1}$, there exists a sequence $\left\{y_{n}\right\} \subset X$ such that $y_{m} \neq y_{n}$ for every $m \neq n$ and $y_{n} \rightarrow x_{1}$. Define a new space $Y$ by identifying $x_{n}$ with $y_{n}$ for every $n>1$ and giving the corresponding quotient topology to $Y$. Then one can verify that $Y$ contains a copy of $S_{2}$ (see the proof of Theorem 2.2) which is not metrizable. So $Y$ is not metrizable. This proves that $(1) \Rightarrow(2)$.

$(2) \Rightarrow(3)$. Suppose $X=A \cup B$ where $A$ is a discrete subspace and $B$ is a compact subspace. We may assume that $A \cap B=\varnothing$.

Claim. $B$ contains $X^{1}$.

On the contrary, if $A \cap X^{1} \neq \varnothing$, say, $x$ is in $A \cap X^{1}$, there exists an open subset $U \subset X$ such that $U \cap A=\{x\}$. Clearly, $\bar{U} \backslash\{x\}$ is a closed subset of $B$ which fails to be compact since $x$ is a limit point of $\bar{U}$. This contradiction proves our claim.

So $X^{1}$ is compact, being a closed subspace of $B$.

$(3) \Rightarrow(1)$. Suppose $X^{1}$ is compact and $f: X \rightarrow Y$ is a quotient map onto $Y$. Denote the set $f\left(X^{1}\right)$ by $Y_{1}$. We use, for convenience, the same symbol $Y_{1}$ to denote the same space as subspace of different spaces. This does not create confusion because the subspace topology is the same in all these cases. $D_{1}$ denotes the set $\left\{x \in X \backslash X^{1}: f(x) \in Y_{1}\right\}$ and $D_{2}$ denotes $\left(X \backslash X^{1}\right) \backslash D_{1}$. Note that $X=X^{1} \bullet D_{1} \bullet D_{2}$ where $\bullet$ denotes the disjoint set union. We denote the set $Y_{1} \bullet D_{1} \bullet D_{2}$ by $X_{1}$ and define a function $g_{1}: X \rightarrow X_{1}$ as follows:

$$
g_{1}(x)= \begin{cases}f(x) & \text { if } x \text { is in } X^{1} \\ x & \text { otherwise }\end{cases}
$$

Provide $X_{1}$ with the quotient topology induced by $g_{1}$. Then we claim that $g_{1}$ is a closed map. For, let $K \subset X$ be a closed subset. Then, $K=K_{1} \bullet K_{2}$ where 
$K_{1} \subset X^{1}$ and $K_{2} \cap X^{1}=\varnothing \cdot g_{1}^{-1}\left(g_{1}(K)\right)=g_{1}^{-1}\left(g_{1}\left(K_{1}\right)\right) \cup K_{2}=g_{1}^{-1}\left(g_{1}\left(K_{1}\right)\right) \cup K$ is closed in $X$ because $g_{1}\left(K_{1}\right)$ is closed in $X_{1}$. Therefore, $g_{1}$ is a closed map. Now by Willard's theorem [7], $X_{1}$ is metrizable. Since $X_{1}^{1} \subset Y^{1}, X_{1}^{1}$ is compact and hence by Theorem 1.1, there exists a normal metric $d$ on $X_{1}$. Now, let $X_{2}$ be the subspace $Y_{1} \bullet D_{2}$ of $X_{1}$. Define $g_{2}: X_{1} \rightarrow X_{2}$ as follows:

$$
g_{2}(x)= \begin{cases}f(x) & \text { if } x \text { is in } D_{1} \\ x & \text { otherwise }\end{cases}
$$

It is not difficult to see that the quotient topology induced by $g_{2}$ and the subspace topology on $X_{2}$ coincide. For every point $y$ in $f\left(D_{2}\right), f^{-1}(y) \subset D_{2}$. We use the same notation $f^{-1}(y)$ as a subset of $X_{2}$ also. Since $d$ is a normal metric, for every $y$ in $f\left(D_{2}\right)$ there exists $x_{y}$ in $f^{-1}(y)$ such that $d\left(x_{y}, Y_{1}\right)<2 d\left(f^{-1}(y), Y_{1}\right)$. Let $D_{3}=\left\{x_{y}: y \in f\left(D_{2}\right)\right\}$ and $X_{3}$ be the subspace $Y_{1} \bullet D_{3}$ of $X_{2}$. We define $g_{3}: X_{2} \rightarrow Y$ as follows:

$$
g_{3}(x)= \begin{cases}f(x) & \text { if } x \text { is in } D_{2} \\ x & \text { otherwise }\end{cases}
$$

For any point $x$ in $X_{3},[x]$ denotes the subset $g_{3}^{-1}\left(g_{3}(x)\right)$ and for any subset $A \subset X_{3}$, $[A]=g_{3}^{-1}\left(g_{3}(A)\right)$. We claim that a subset $F \subset X_{3}$ is closed in $X_{3}$ if and only if. $[F]$ is closed in $X_{2}$. Since $[F] \cap X_{3}=F, F$ is closed in $X_{3}$ whenever $[F]$ is closed in $X_{2}$. Now, suppose $F$ is closed in $X_{3}$ and $x$ is in $\overline{[F]}$. To show that $x$ is in $[F]$. Note that $x$ is in $[F]$ if $x$ is an isolated point in $\overline{[F]}$. So we assume that $x$ is not an isolated point in $\overline{[F]}$. Observe that $x$ is in $Y_{1}$. If every neighborhood of $x$ meets $[F] \cap Y_{1}=F \cap Y_{1}$ at infinitely many points, then $x$ is in $\overline{F \cap Y_{1}}=F \cap Y_{1}$ (because $Y_{1}$ is compact and $F$ is closed) $\subset[F]$. Suppose $x$ has a neighborhood $U_{x}$ such that $U_{x} \cap Y_{1}=\{x\}$. For a given $\varepsilon>0$, let $B_{\varepsilon}(x)$ denote the $\varepsilon$-ball around $x$ in $X_{2}$. Choose $x^{\prime}$ in $B_{\varepsilon}(x) \cap U_{x}$ (which is infinite) such that $x \neq x^{\prime}$ and $d\left(x^{\prime}, x\right)<\varepsilon / 2$. Then by the choice of $D_{3}, d\left(x_{f\left(x^{\prime}\right)}, x\right)<\varepsilon$ and therefore $x_{f\left(x^{\prime}\right)}$ is in $B_{\varepsilon}(x)$. Hence $x_{f\left(x^{\prime}\right)}$ is in $B_{\varepsilon}(x) \cap F$, showing that $B_{\varepsilon}(x) \cap F$ is nonempty for all $\varepsilon$. So $x$ is in $\bar{F}=F \subset[F]$. This completes the proof that $[F]$ is also closed whenever $F$ is closed.

Now, let $h$ denote the restriction of $g_{3}$ to $X_{3}$. We claim that $h: X_{3} \rightarrow Y$ is a homeomorphism. $h$ is one-one and onto. Let $F$ be closed in $Y$. Then $f^{-1}(F)$ is closed in $X$ and saturated with respect to $g_{2} \circ g_{1}$. So $g_{2}\left(g_{1}\left(f^{-1}(F)\right)\right)$ is closed in $X_{2}$ and hence $h^{-1}(F)=g_{2}\left(g_{1}\left(f^{-1}(F)\right)\right) \cap X_{3}$ is closed in $X_{3}$. Thus $h$ is continuous. If $[F]$ is closed in $X_{2}$, then $g_{1}^{-1}\left(g_{2}^{-1}([F])\right)$ is closed in $X$. Since $g_{1}^{-1}\left(g_{2}^{-1}([F])\right)$ is saturated with respect to $f, f\left(g_{1}^{-1}\left(g_{2}^{-1}([F])\right)\right)=h(F)$ is closed in $Y$. Hence $h$ is a homeomorphism. This proves that $Y$ is metrizable because $X_{3}$ is.

$(3) \Rightarrow(4)$. Suppose $X^{1}$ is compact. If $\omega^{2}$ is cmbedded in $X$ as a closed subspace, then $\{\omega . n: n \in \mathbf{N}\}$ is an infinite discrete closed subspace of $X^{1}$. This contradicts the hypothesis that $X^{1}$ is compact and thus $(3) \Rightarrow(4)$ is proved.

$(4) \Rightarrow(3)$ can be proved by a reverse argument of the proof of $(3) \Rightarrow(4)$.

2. Some more equivalent forms. In this section, we give many more equivalent forms of the statements of Theorem 1.2. A topological space $Y$ is called a Fréchet space if for any subset $A \subset Y, y$ is in $\bar{A}$ if and only if there exists a sequence in $A$ converging to $y$. We exploit properties of $S_{2}$ to obtain many more equivalent forms of the property that "every quotient of the space is metrizable". 
THEOREM 2.1. For a metric space $X$, the following conditions are equivalent:

(a) Every quotient of $X$ is metrizable.

(b) Every quotient of $X$ is first countable.

(c) Every quotient of $X$ is a Fréchet space.

Statement (a) is equivalent to any of the following:

(d) every compact-to-one quotient of $X$ is $P$,

(e) every finite-to-one quotient of $X$ is $P$,

(f) every $n$-to-one quotient of $X$ is $P$ for all $n$ in $\mathbf{N}$,

where $P$ stands for the property of being either 'metrizable' or 'first countable' or 'a Fréchet space'.

Every statement obtained by replacing 'quotient' by 'open continuous image' in statements (b) to (f) is again equivalent to (a).

PROOF. The proof follows from the following facts:

(i) $S_{2}$ does not satisfy $P$ (where $P$ is as in the statement) [3].

(ii) We can find an open, two-to-one, continuous image of $X$ containing $S_{2}$ whenever $X^{1}$ is not compact (see the space $Y$ in the proof of $(3) \Rightarrow(2)$ of Theorem 2.2).

(iii) Metrizable $\Rightarrow$ first countable $\Rightarrow$ a Fréchet space [3].

A quotient map $q: X \rightarrow Y$ onto $Y$ is called hereditarily quotient if the restriction $\left.q\right|_{A}$ of $q$ to $A \subset X$ is again a quotient map for every saturated subspace $A$ of $X$. $q$ is called pseudo-open if $y$ is in $\operatorname{Int} q(U)$ for every open set $U$ containing $q^{-1}(y)$.

Arkhangel'skii [1] has proved that a quotient map is hereditarily quotient if and only if it is pseudo-open. See also [4].

THEOREM 2.2. On a metric space $X$ the following conditions are equivalent:

(1) Every quotient of $X$ is metrizable.

(2) Every surjective quotient map from $X$ is pseudo-open.

(3) Every surjective quotient map from $X$ is hereditarily quotient.

PROOF. (1) $\Rightarrow(2)$. By Theorem 1.2, (1) implies that $X^{1}$ is compact. Let $f: X \rightarrow Y$ be a surjective quotient map and $D=X \backslash f^{-1}\left(f\left(X^{1}\right)\right)$. Denote by $X_{1}$ the set $D \cup f\left(X^{1}\right)$ and define $g: X \rightarrow X_{1}$ as follows:

$$
g(x)= \begin{cases}x & \text { if } x \text { is in } D \\ f(x) & \text { otherwise }\end{cases}
$$

Providing $X_{1}$ with the quotient topology induced by $g$, one can prove that $g$ is a closed map by a similar argument as in the proof of Theorem 1.2. Define $h: X_{1} \rightarrow Y$ as follows:

$$
h(x)= \begin{cases}f(x) & \text { if } x \text { is in } D, \\ x & \text { otherwise. }\end{cases}
$$

Then $h$ is an open map onto $Y$ and $f=h \circ g$. Since both open maps and closed maps are pseudo-open, we can prove that $f$ is pseudo-open.

$(2) \Rightarrow(3)$ is known $[\mathbf{1}]$.

$(3) \Rightarrow(1)$. If $X$ does not satisfy (1), $X^{1}$ is not compact by Theorem 1.2 . So we can choose an infinite discrete closed subset $\left\{x_{m}: m=0,1,2, \ldots\right\}$ of $X^{1}$. Let $\left\{U_{m}: m=0,1,2, \ldots\right\}$ be a sequence of pairwise disjoint open subsets of $X$ such 
that $x_{m}$ is in $U_{m}$ for all $m$. For each $m=0,1,2, \ldots$, we can choose a sequence $\left\{x_{m, n}: n \in \mathbf{N}\right\} \subset U_{m}$ such that $x_{m, n}$ converges to $x_{m}$. Let

$$
Y=X \backslash\left\{x_{n}: n=1,2, \ldots\right\} \text { and } f: X \rightarrow Y
$$

be defined as follows:

$$
f(x)= \begin{cases}x_{0, n} & \text { if } x=x_{n} \\ x & \text { otherwise }\end{cases}
$$

Give quotient topology for $Y$ (induced by $f$ ).

Claim. $f$ is not hereditarily quotient.

For, consider $A=\left\{x_{m, n}: m, n=1,2, \ldots\right\} \cup\left\{x_{0}\right\} . A$ is saturated. If $\left.f\right|_{A}$ were a quotient map onto its image $f(A)$, then $f\left(x_{0}\right)$ would have been isolated in $f(A)$. But $f\left(x_{0}\right)$ is not isolated in $f(A)$. Thus the claim is proved. This proves $(3) \Rightarrow(1)$.

REMARKS. 1. The statements (2) and (3) of Theorem 2.2 have some more equivalent forms similar to those of Theorem 2.1. Each statement obtained by replacing 'quotient map' in (2) and (3) by 'compact-to-one quotient map', finiteto-one quotient map' etc. is equivalent to statement (1). Further the statements obtained by replacing 'quotient maps' by 'open maps' in all the above cases are also equivalent to (1).

2. In [4], the author has obtained a necessary and sufficient condition for every quotient of a space to be hereditarily quotient. The reader shall note that our conditions are entirely different from what is obtained in [4] because we consider quotients in only Hausdorff spaces in the present article.

3. Arkhangel'skii discusses three general problems in his survey article [1]. The first among them is the following: Under what circumstances can each space of a given class $A$ be mapped onto a space of a given class $B$ by means of a mapping belonging to a given class $L$ ? When this is possible he writes $A L B$. Here we have found a maximal subclass $A$ of the class of all metric spaces in connection with this problem for different classes $L$ of maps such as open maps, quotient maps, finite-to-one quotient maps, hereditarily quotient maps etc., and for classes $B$ such as metric spaces, first countable spaces and Fréchet spaces and showed that $A L B$ holds.

\section{REFERENCES}

1. A. V. Arkhangel'skii, Mappings and spaces, Russian Math. Surveys 21 (1966), 115-162.

2.

3. A. V. Arkhangel'skii and S. P. Franklin, Ordinal invariants for topological spaces, Michigan Math. J. 15 (1968), 313-320.

4. V. Kannan, Ordinal invariants in topology, Mem. Amer. Math. Soc. No. 245 (1981).

5. S. G. Mrowka, Normal metrics, Amer. Math. Monthly 72 (1965), 998-1001.

6. A. H. Stone, Metrizability of decomposition spaces, Proc. Amer. Math. Soc. 7 (1956), 690-700.

7. S. Willard, Metric spaces all of whose decompositions are metric, Proc. Amer. Math. Soc. 21 (1969), 126-128.

8. _ General topology, Addison-Wesley, 1970.

SChool of Mathematics \& COMPUter/INFormation SCIENCES, UNiversity of HYDERABAD, HYDERABAD 500 134, INDIA 